\title{
Audit and feedback: to tell or not to tell? That is not the question
}

\author{
Simon Whyte, MBBS, FRCA, FRCPC@
}

Received: 23 July 2018/Accepted: 24 July 2018/Published online: 22 August 2018

(C) Canadian Anesthesiologists' Society 2018

In the interests of improving patient safety and quality of care, physicians have, in recent years, been encouraged to embrace reflective practices that include receiving and responding to feedback on aspects of performance. An extensive body of literature provides education, advice, and some evidence on what to measure, including how and how often to do so. ${ }^{1}$ Much debated is how best to provide these data to physicians in a way that will be received as being constructive, non-punitive, and most likely to engage them in using the information to change practices that may improve patients' experiences and outcomes. ${ }^{2}$

In this issue of the Journal, ${ }^{3}$ Boet et al. report the findings from their study of the effect of audit and feedback on anesthesiologists' intraoperative temperature management and patient outcomes. They found that providing benchmarked or ranked feedback of audit data was no better than providing no feedback at all when it came to improving the incidence of intraoperative hypothermia.

I imagine the findings of this study will surprise some and disappoint many. The existing body of evidenceextensively cited in the authors' paper-suggests that feedback usually works, but their study suggests the opposite. What should we do now? As always, we should carefully examine the study's methodology, findings, and conclusions. In doing so, we will learn that the findings are not as surprising or unexpected as we might instinctively have thought.

S. Whyte, MBBS, FRCA, FRCPC ( $\square)$

Department of Anesthesia, British Columbia's Children's

Hospital, University of British Columbia, Vancouver, BC, Canada

e-mail: swhyte@cw.bc.ca
There are a number of methodologic considerations to highlight in this generally well-conducted study. Boet et al obtained consent from 45 anesthesiologists to audit three metrics: their use of forced air warming, patients' temperatures on arrival in the postanesthesia care unit (PACU), and their pass/fail rate in compliance with a definition of good perioperative temperature management taken from the Physician Quality Reporting System (PQRS; now called the Merit-based Incentive Payment System). ${ }^{4}$ These audit data were not initially fed back, but the participants knew they were being collected. This eight-month time window thus constituted a baseline period that could potentially have been influenced by a Hawthorne effect- i.e., the participants may have altered their behaviour simply as a result of knowing that it was being evaluated in some way. Following this baseline period of audit without feedback, the anesthesiologists were cluster-randomized into one of three groups. The control group continued to receive no feedback on their audit data. The "benchmarked feedback" group received monthly feedback on whether or not their patients' incidence of hypothermia was below a predetermined target incidence (deemed to be an acceptable standard of care for this metric). The "ranked feedback" group received monthly feedback on where they ranked compared with other anesthesiologists in the department (as measured by their own patients' incidence of hypothermia).

When we look at the audit data from the baseline period, we learn a number of important facts that influence how we should interpret the subsequent effects of feedback. The baseline incidence of hypothermia was $25 \%$ (not $50 \%$ as was assumed a priori for the sample size calculations); the baseline use of forced air warming was $85-88 \%$; and the baseline pass rate for compliance with the PQRS standard 
was $100 \%$. Given that a quarter of all patients were hypothermic on arrival in PACU, despite nearly nine of ten already having received intraoperative forced air warming, it was entirely predictable that this outcome metric was unlikely to be improved by telling (or not telling) anesthesiologists how often they employed forced air warming. It should be noted that both types of feedback actually did positively impact the process metric, as use of forced air warming increased to $94 \%$ in the feedback groups. And of course, there was no scope for improvement in the PQRS compliance metric.

The authors reported that, of 75 anesthesiologists approached, 45 consented to participate. Nevertheless, the 15 subjects randomized to receive ranked feedback appeared to have been ranked using a denominator of 79 (comprising the 45 consenting participants, the 30 who did not consent and thus on whom no data were collected, and the four study investigators who are also department members). This may matter because, for example, being told that one ranked $20^{\text {th }}$ of $79 v s 20^{\text {th }}$ of 45 might provide different motivations to change performance. Additionally, if I was told I was one of the top-performing anesthesiologists in my group, I might be rather pleased-but would be less so if I then learned that I had failed to meet the expected target ("standard of care"). Conversely, depending on personality characteristics, one can imagine that some people would be content just to know that they were exceeding benchmark expectations, and might be less bothered by their rank. The point is that the two methods of feedback may be complementary and there may be a role for both. This was not examined in this study. Finally, given that everyone had a $100 \%$ "pass" rate on PQRS, this may have negatively affected motivation to change when provided with feedback. It would have been interesting to conduct a post-study assessment of the impact of feedback on the study participants.

The authors concluded that the "study found no evidence to suggest that a formative assessment using benchmarked or ranked feedback was more effective than no feedback to influence anesthesiologists' performance on patient temperature outcome in our clinical setting". ${ }^{3}$ In reality, the truth may be a little more nuanced. While they found no evidence that feedback on intraoperative temperature management reduced the incidence of hypothermia, they did find clear evidence of altered performance-i.e., warmer use increased in all three groups. Another way of interpreting their findings is that feedback did not further improve any Hawthorne effect that had already been achieved-i.e., simply being audited was sufficient to incentivize all participants to maximally improve their performance. As the authors note, other investigators have failed to show the impact of intraoperative forced air warming on PACU arrival temperature. Of interest is that the feedback included a number of tips on how to optimize perioperative thermoregulation, but preoperative warming-which occurred in only about one third of cases, and which is associated with reduced intraoperative hypothermia load and reduced allogeneic transfusion ${ }^{5,6}$ - was not highlighted. This perhaps represents a missed opportunity, even though it is a process of care that is not always directly under the anesthesiologist's control.

Quality of care is a multi-faceted construct and has classically been described in the Institute of Medicine's (IoM) 2000 publication "To Err Is Human"7 as comprising six pillars or domains: safety, timeliness, efficiency, costeffectiveness, equity, and patient-centerdness. The Donabedian model $^{8}$ provides a framework for evaluating quality of healthcare within any of these domains, by examining (infra)structures, processes, and outcomes of importance. A moment's consideration reveals a bewildering complexity of permutational interactions, both within and between IoM domains, and in the relationships between structures, processes, and relevant outcomes in healthcare. Deciding which outcomes are relevant, or how to prioritize competing interests, may depend on one's perspective: patient, family member, physician, administrator, to name a few.

Boet et al.'s study illustrates a common challenge for quality improvement in anesthesia-process changes (in this instance, increased utilization of intraoperative forced air warming) do not always result in outcome changes (in this case, less hypothermia in PACU). The relationship between process improvement and outcome improvement is complex and not necessarily tightly linked. Many other factors may confound it-either by being more important or by mitigating/offsetting. The more temporally remote an outcome is from an intervention that is postulated to contribute to that outcome, the harder it becomes to isolate, attribute, and quantify any hypothesized contribution. Thus, just because improving a process does not directly improve the outcome, it does not mean that process improvement is not valuable. It may be necessary, but not sufficient. Patients, and the environment in which we care for them, are highly complex systems. We should not be surprised or disheartened when a change that we implement, which we believe to be relevant and logical, does not immediately result in the hoped-for gain. The authors recognize this and allude to the possible need for an improvement bundle, wherein several process changes may need to be effectively and reliably implemented to generate a measurable impact on outcome.

Another fundamental challenge in quality improvement science is choosing what to measure. Measurement is clearly critical to the basic paradigms of quality improvement methodologies, such as LEAN, Six-Sigma, 
and $\mathrm{PDSA}^{9,10}$ - these all require us to measure something, a yardstick against which we can evaluate our attempts to improve. Choosing good metrics is easier said than done because healthcare is complicated, and measuring "complicated" is complicated! Metrics that are simple to measure, but aim to summarise complexity, are somewhat of a double-edged sword, as they do not reduce the complexity and they may hide or even create problems. Relevant examples of this dilemma of using summary measures to quantify domains of care quality include firstcase start times, wait list times and, yes, even temperature measurement. Does a single non-core temperature measurement on arrival in the PACU truly reflect perioperative management of thermoregulatory homeostasis? Is $36^{\circ} \mathrm{C}$ the right cut-off? Would a measure of intraoperative duration or severity of hypothermia-or both-be a better metric, though at the expense of being more difficult to measure? And so on.

Boet $e t$ al. have reported a methodologically challenging study that gives all of us plenty to think about. As good studies often do, it raises more questions than it necessarily answers. In the ongoing search for causality between what we do and how patients do as a result, audit and feedback generally improve our performance and improve our patients' outcomes. But the two will not always walk in lockstep and we anesthesiologists may have to be more patient than our instant-gratification personalities may be accustomed to.

\section{Audit et rétroaction: dire ou ne pas dire? Telle n'est pas la question}

Dans l'intérêt de l'amélioration de la sécurité des patients et de la qualité des soins dans les dernières années, les médecins ont été encouragés à adopter des pratiques réflectives incluant la réception et la réponse à une rétroaction sur des aspects de leurs performances. Un grand corpus de publications procure de la formation, des conseils et quelques données probantes sur ce qu'il y a lieu de mesurer et sur comment et à quelle fréquence le faire. ${ }^{1}$ La question la plus débattue est de savoir comment fournir aux médecins ces données d'une manière qui sera constructive et ne sera pas ressentie comme punitive, mais aussi aura le plus de chances de les inciter à utiliser cette information pour modifier leurs pratiques afin d'améliorer dans la mesure du possible l'évolution et l'expérience des patients. ${ }^{2}$

Dans ce numéro du Journal, Boet et coll. rapportent les constatations de leur étude sur l'effet d'une enquête et de la rétroaction sur la gestion de la température peropératoire par les anesthésiologistes et l'évolution des patients. Ils ont trouvé que fournir une rétroaction par performance ou classement sur les données de l'enquête n'était pas supérieur à l'absence de rétroaction quand il s'agissait d'améliorer l'incidence de l'hypothermie peropératoire.

J'imagine que ces constatations en surprendront quelques-uns et en décevront plusieurs. L'ensemble des données probantes existantes - abondamment citées dans l'article de ces auteurs - laisse penser qu'une rétroaction est habituellement efficace, mais leur étude porte à croire le contraire. Que doit-on faire maintenant? Comme toujours, nous devons analyser attentivement la méthodologie, les constatations et les conclusions de l'étude. Ainsi, nous apprendrons que les constatations ne sont pas si surprenantes ni si inattendues que nous l'aurions instinctivement pensé.

Il y a plusieurs questions de méthodologie à souligner dans cette étude globalement bien faite. Boet et coll. ont obtenu le consentement de 45 anesthésiologistes pour vérifier trois paramètres: leur utilisation du réchauffement à air pulsé, la température des patients à leur arrivée dans la salle de réveil et leur taux de succès/ échec en matière de conformité par rapport à la bonne gestion de la température périopératoire (extraite du Système médical de déclaration de la qualité PQRS; maintenant appelé Système de rémunération au mérite). ${ }^{4}$ Ces données de vérification n'avaient pas donné initialement lieu à une rétroaction, mais les participants savaient qu'elles étaient collectées. Cette fenêtre de huit mois a donc constitué une période de référence qui aurait donc pu être influencée par un «effet Hawthorne », c'està-dire que les participants peuvent avoir modifié leur comportement simplement parce qu'ils savaient qu'ils faisaient l'objet d'une évaluation quelconque. À la suite de cette période de référence de vérification sans rétroaction, les anesthésiologistes ont été randomisés par grappes (clusters) dans trois groupes. Le groupe témoin a continué à ne recevoir aucune rétroaction sur ses données vérifiées. Le groupe de « rétraction sur la performance » a reçu une rétroaction mensuelle indiquant si l'incidence d'hypothermie de ses patients était (ou non) inférieure à une cible préétablie et considérée comme étant une norme de soins acceptable pour ce paramètre. Le groupe de « rétroaction par classement» a reçu une rétroaction mensuelle indiquant où ils se situaient par rapport aux autres anesthésiologistes du département sur l'incidence d'hypothermie de leurs patients.

Quand nous examinons les données de vérification de la période de référence, nous découvrons un certain nombre de points importants qui ont une influence sur comment nous devons interpréter les effets subséquents de la rétroaction. L'incidence initiale de l'hypothermie était de 
$25 \%$ et non de $50 \%$ comme supposé a priori pour les calculs de taille de l'échantillon; l'utilisation initiale du réchauffement par air pulsé était de $85 \%$ à $88 \%$ et le taux de passage initial de conformité avec la norme PQRS était de $100 \%$. Considérant qu'un quart de tous les patients était hypothermique à l'arrivée en salle de réveil, bien que neuf sur dix parmi eux aient bénéficié d'un réchauffement à air pulsé peropératoire, il était absolument prévisible que ce paramètre avait peu de chances d'être amélioré en informant (ou en n'informant pas) les anesthésiologistes de la fréquence à laquelle ils avaient employé un réchauffement à air pulsé. Il faut noter que les deux types de rétroaction ont eu effectivement un impact positif sur le paramètre puisque l'utilisation du réchauffement a air pulsé a augmenté à $94 \%$ dans les deux groupes de rétroaction. Et, naturellement, il n'y avait pas de possibilité d'amélioration concernant la mesure de conformité au PQRS.

Les auteurs ont indiqué que sur les 75 anesthésiologistes qui avaient été approchés, 45 avaient consenti à participer. Néanmoins, les 15 participants randomisés pour recevoir une rétroaction par classement semblent avoir été classés avec un dénominateur de 79 incluant les 45 participants consentants, les 30 n'ayant pas consenti (et pour lesquels aucune donnée n'était collectée) et les quatre investigateurs de l'étude qui sont également des membres des départements. Cela pourrait être important parce que, par exemple, apprendre que l'on est classé $20^{\mathrm{e}}$ sur 79 au lieu de $20^{\mathrm{e}}$ sur 45 peut créer des motivations différentes à un changement de performance. De plus, si on me disait que j'étais l'anesthésiologiste le plus performant de mon groupe, je serais plutôt satisfait, mais je le serais moins si j'apprenais alors que je n'avais pas réussi à atteindre la cible attendue (la «norme de soins de référence »). À l'opposé, en fonction des caractéristiques de la personnalité, on peut imaginer que certaines personnes seraient juste heureuses de savoir qu'elles dépassaient les attentes de performance et beaucoup moins contrariées par leur classement. Le point important est que les deux méthodes de rétroaction pourraient être complémentaires et que les deux peuvent avoir un rôle à jouer. Cela n'a pas été examiné dans l'étude. Enfin, compte tenu du fait que tout le monde avait un taux de «réussite » de $100 \%$ au PQRS, cela peut avoir eu des répercussions négatives sur la motivation à changer, suite à la rétroaction. Il aurait été intéressant de mener une évaluation post étude sur l'impact de la rétroaction sur les participants à l'étude.

Les auteurs ont conclu que «l'étude n'avait trouvé aucune donnée probante suggérant qu'une évaluation formative utilisant une rétroaction par performance ou classement était plus efficace que l'absence de rétroaction pour influencer la performance des anesthésiologistes sur la température des patients dans notre cadre clinique ». ${ }^{3}$ En réalité, la vérité doit être un peu plus nuancée. Bien qu'ils n'aient pas trouvé de données probantes montrant que la rétroaction sur la gestion peropératoire de la température réduisait l'incidence de l'hypothermie, ils ont trouvé des données évidentes montrant un changement de performance, à savoir que l'utilisation du réchauffement avait augmenté dans les trois groupes. Une autre interprétation de leurs constatations était que la rétroaction n'amenait pas d'amélioration par rapport à ce qui avait été déjà obtenu par l'effet Hawthorne, c'est-à-dire que d'être soumis à une vérification était un incitatif suffisant pour l'ensemble des participants à améliorer leurs performances au maximum. Comme le notent les auteurs, d'autres investigateurs ne sont pas parvenus à montrer l'impact du réchauffement peropératoire à air pulsé sur la température à l'arrivée en salle de réveil. Il est intéressant de noter que la rétroaction incluait plusieurs conseils sur la façon d'optimiser la thermorégulation, mais que le réchauffement préopératoire (qui n'a été réalisé que dans environ un tiers des cas et qui est associé à une moindre charge hypothermique peropératoire et à une réduction des transfusions allogéniques) $)^{5,6}$ n'était pas mis en évidence. Ceci constitue peut-être une opportunité manquée, même s'il s'agit d'un processus de soins qui n'est pas toujours directement sous le contrôle de l'anesthésiologiste.

La qualité des soins est un ensemble à multiples facettes qui a été classiquement décrit en 2000 dans une publication de l'Institute of medecine (IoM, États-Unis) intitulée « L'erreur est humaine ${ }^{7}$ comme reposant sur six piliers ou domaines : innocuité, moment opportun, efficience, rapport coût-efficacité, équité et soins axés sur le patient. Le modèle de Donabedian ${ }^{8}$ procure un cadre d'évaluation de la qualité des soins de santé au sein de chacun de ces domaines en examinant les (infra)structures, les processus et les résultats importants. Une réflexion rapide révèle une incroyable complexité d'interactions permutées, à la fois au sein de chaque domaine et entre les domaines de l'IoM, et dans les rapports entre les structures, processus et résultats pertinents pour les soins de santé. Décider quels points d'aboutissement sont pertinents ou comment classer par ordre de priorité des intérêts conflictuels dépend du point de vue de chacun : patient, membre de la famille, médecin, administrateur, pour n'en citer que quelques-uns.

L'étude de Boet et coll. illustre quelques-uns des défis courants de l'amélioration de la qualité en anesthésie; les changements de processus (dans le cas présent, une plus grande utilisation du réchauffement peropératoire à air pulsé) n'entraînent pas toujours des changements dans les résultats (ici, un moins grand nombre d'hypothermies en salle de réveil). Les rapports entre l'amélioration des processus et celle des résultats sont complexes et pas nécessairement étroitement liés. Il peut y avoir de nombreux autres facteurs confondants, possiblement plus 
importants ou réduisant/annulant les résultats. Plus un résultat est distant dans le temps de l'intervention supposée y avoir contribué, plus il devient difficile d'isoler, attribuer et quantifier une contribution hypothétique. Ainsi, ce n'est pas parce que l'amélioration d'un processus n'améliore pas directement le résultat que le processus d'amélioration n'est pas valable. Cela peut être nécessaire, mais pas suffisant. Les patients et l'environnement dans lequel nous prenons soin d'eux sont des systèmes d'une grande complexité. Nous ne devrions donc pas être surpris ou découragés quand le changement que nous avons mis en œuvre, car nous paraissant pertinents et logiques, ne donne pas immédiatement les bénéfices escomptés. Les auteurs l'admettent et font allusion au besoin possible d'un ensemble d'améliorations simultanées, où plusieurs changements de processus pourraient être devoir être implémentés de manière efficace et fiable pour générer un impact mesurable sur le résultat.

Un autre défi fondamental de la science de l'amélioration de la qualité est de choisir quoi mesurer. Mesurer est quelque chose d'absolument essentiel dans les paradigmes des méthodologies d'amélioration de la qualité, tels que LEAN, Six-Sigma et PDSA (planifier, faire, étudier, agir) ${ }^{9,10}$ : tous exigent que nous mesurions quelque chose, un étalon contre lequel nous pouvons évaluer nos tentatives d'amélioration. Choisir les bons paramètres à mesurer est plus facile à dire qu'à faire parce que les soins de santé sont compliqués et que mesurer quelque chose de « compliqué » est... compliqué! Des paramètres qui sont simples à mesurer, mais visent à résumer la complexité, sont en quelque sorte des armes à double tranchant, car ils ne peuvent pas réduire la complexité et ils peuvent cacher ou même créer des problèmes. Les exemples pertinents de ce dilemme de l'utilisation de mesures synthétiques pour quantifier des domaines de qualité des soins incluent les délais de démarrage des premiers cas, les délais des listes d'attente et — oui — même la mesure de la température. Est-ce qu'une seule mesure de la température (non centrale) à l'arrivée en salle de réveil reflète véritablement la gestion périopératoire de l'homéostasie thermorégulatoire? Le seuil correct est-il de $36{ }^{\circ} \mathrm{C}$ ? Une mesure de la durée peropératoire ou de la sévérité de l'hypothermie, ou des deux, constituerait-elle un meilleur paramètre de mesure, bien qu'étant plus difficile à mesurer ? Et ainsi de suite.

Boet et coll. ont rapporté une étude méthodologiquement provocatrice qui nous donne beaucoup à réfléchir. Comme le font souvent les bonnes études, elle soulève plus de questions qu'elle n'apporte de réponses. Dans la quête continue de causalité entre ce que nous faisons et ce que comment les patients évoluent en conséquence, la vérification et la rétroaction améliorent habituellement nos performances et l'évolution de nos patients. Mais les deux n'iront pas toujours de pair et, en tant qu'anesthésiologistes, nous pourrions devoir être plus patients que nos personnalités accoutumées à une gratification immédiate n'y sont habituées.

Conflicts of interest None declared.

Editorial responsibility This submission was handled by Dr. Hilary P. Grocott, Editor-in-Chief, Canadian Journal of Anesthesia.

Conflits d'intérêts Aucun déclaré.

Responsabilité éditoriale Cet article a été traité par le $\mathrm{D}^{\mathrm{r}}$ Hilary $\mathrm{P}$. Grocott, Rédacteur en chef, Journal canadien d'anesthésie.

\section{References}

1. Wagstaff DT, Bedford J, Moonesinghe SR. Improvement science in anaesthesia. Curr Anesthesiol Rep 2017; 7: 432-9.

2. D'Lima D, Arnold G, Brett SJ, Botte A, Smith A, Benn J. Continuous monitoring and feedback of quality of recovery indicators for anaesthetists: a qualitative investigation of reported effects on professional behaviour. Br J Anaesth 2017; 119: 11524.

3. Boet S, Bryson GL, Taljaard M, et al. Effect of audit and feedback on physicians' intraoperative temperature management and patient outcomes: a three-arm cluster randomized-controlled trial comparing benchmarked and ranked feedback. Can J Anesth 2018; 65. DOI: https://doi.org/10.1007/s12630-018-1205-0

4. Centers for Medicare and Medicaid Services. This website contains a link, under MIPS/Quality/Claims Registry Measures 401-467, to MIPS \#424, which details the relevant standard as a pdf download. Available from URL: https://www.cms.gov/ Medicare/Quality-Payment-Program/Resource-Library/2018Resources.html (accessed July 2018).

5. Görges M, Ansermino JM, Whyte $S D$. A retrospective audit to examine the effectiveness of preoperative warming on hypothermia in spine deformity surgery patients. Paediatr Anaesth 2013; 23: 1054-61.

6. Görges $M$, West NC, Cheung W, Zhou G, Miyanji F, Whyte SD. Preoperative warming and undesired surgical and anesthesia outcomes in pediatric spinal surgery-a retrospective cohort study. Paediatr Anaesth 2016; 26: 866-75.

7. Institute of Medicine. (US) Committee on Quality of Health Care in America; Kohn LT, Corrigan JM, Donaldson MS. To Err is Human: Building a Safer Health. System. 2000; DOI: https://doi. org/10.17226/9728.

8. Donabedian A. The quality of care. How can it be assessed? JAMA 1988; 260: 1743-8.

9. Adams D. Quality improvement; part 1: introduction and overview. BJA Education 2018; 18: 89-94.

10. Varughese AM, Rampersad SE, Whitney GM, Flick RP, Anton B, Heitmiller ES. Quality and safety in pediatric anesthesia. Anesth Analg 2013; 117: 1408-18. 